\title{
Nonequilibrium Phase Transitions of Impact Ionization Breakdown in Extrinsic Semiconductors
}

\author{
J. Parisi ${ }^{1,2}$, J. Peinke $^{1}$, U. Rau ${ }^{1}$, and W. Clauß ${ }^{1}$ \\ 1 Physikalisches Institut, Lehrstuhl Experimentalphysik II, Universität Tübingen, Tübingen, FRG \\ 2 Physikalisches Institut, Lehrstuhl Experimentalphysik V, Universität Bayreuth, Bayreuth, FRG
}

Z. Naturforsch. 45a, $1048-1050$ (1990); received May 23, 1990

In the study of semiconductor electronic breakdown we observe the self-generated formation of spatio-temporal dissipative structures, when a bias voltage is applied at liquid-helium temperature. The underlying nonlinear physics of impurity impact ionization reveals critical phase transition behavior by varying the temperature at constant voltage.

Key words: Semiconductor breakdown, Structure formation, Critical phase transition

\section{Introduction}

A complex system may consist of many subsystems. The dimension of the equations used to model such a system may be enormously large. It is well kown that a nonequilibrium complex system may undergo a variety of bifurcations and exhibit diverse types of self-organization behavior. It was found, however, that in the vicinity of a bifurcation point the enormous number of subsystems is governed by a few coherent variables which force the subsystems to join a collective motion. Those coherent variables are often called order parameters. The dimension of the order parameters is much smaller than that of the initial complex system. The underlying slaving principle elucidates how, after the competition of various modes, a few coherent order parameters arise beyond the bifurcations to complex space- and time-dependent configurations and how the many subsystems, or say variables, are controlled (or, enslaved) by them. Such ubiquitous processes of spontaneous self-organization can in general be formulated as nonequilibrium orderdisorder phase transitions [1].

In this presentation, we discuss the application of the universal approach outlined above to nonlinear transport phenomena in semiconductors providing a prototype situation with dynamical behavior ranging from orderly to chaotic [2]. In particular, recent experiments on impurity impact ionization avalanche breakdown in extrinsic germanium and gallium arsenide at

Reprint requests to Priv.-Doz. Dr. Jürgen Parisi, Physikalisches Institut, Lehrstlihl Experimentalphysik II, Universität Tübingen, Morgenstelle 14, D-7400 Tübingen, FRG. low temperatures have demonstrated the self-sustained development of both filamentary spatial and oscillatory temporal dissipative structures in the formerly homogeneous bulk semiconductor [3-7]. This kind of nonequilibrium phase transition between different conducting states results from the autocatalytic nature of impurity impact ionization generating mobile charge carriers [8]. The simple and direct experimental accessibility via advanced measurement techniques favors semiconductors as a nearly ideal study object for complex nonlinear dynamics compared to the other physical systems [9]. Further representing a convenient reaction-diffusion system that exhibits distinct universal features, the present semiconductor system may receive general significance for many synergetic systems in nature [10]. Finally, in view of the rapidly growing application of semiconductor technologies, the understanding, control, and possible exploitation of sources of instability in these systems have considerable practical importance.

\section{Physics}

As an exemplary experimental system, we focus in the following on single-crystalline p-doped germanium, displaying spatio-temporal nonlinear current flow when electrically driven into low-temperature impact ionization breakdown. Having the typical dimensions of about $(0.2 \times 2 \times 5) \mathrm{mm}^{3}$ and an indium acceptor concentration of about $10^{14} \mathrm{~cm}^{-3}$, the extrinsic germanium crystal carries properly arranged ohmic aluminum contacts evaporated on one of the two largest surfaces. To provide the ohmic contacts 
with an electric field, a d.c. bias voltage was applied to the series combination of the sample and a load resistor. The resulting current was found from the voltage drop at the load resistor. Utilizing low-temperature scanning electron microscopy, two-dimensional images of both stationary and dynamical current structures were obtained by scanning the specimen surface with an electron beam chopped properly and by recording the beam-induced current change in the voltage-biased sample as a function of the beam coordinate. The present imaging method combines a lowtemperature stage with a commercial scanning electron microscope such that both spatial and temporal measurements can be performed simultaneously. During the experiments, the semiconductor sample was always kept at liquid-helium temperatures (less than $10 \mathrm{~K}$ ) and carefully protected against external electromagnetic irradiation $[3,5]$.

Analogous to the corresponding processes of structure formation in gaseous plasma discharges and atmospheric lightning, impact ionization of the shallow impurity acceptors can be achieved in the bulk of the homogeneously doped semiconductor at low temperatures. In the temperature regime of liquid helium, most of the charge carriers are frozen out at the impurities. Since the ionization energy is only about $10 \mathrm{meV}$ and electron-phonon scattering is strongly reduced, avalanche breakdown due to an abrupt multiplication of mobile charge carriers already takes place at electric fields of a few $\mathrm{V} / \mathrm{cm}$ and persists until nearly all impurities are ionized [11]. The underlying nonequilibrium phase transition from a low conducting state to a high conducting state is directly reflected in strongly nonlinear regions of negative differential resistivity in the microscopic current-density versus electric-field characteristic. Accordingly, the autocatalytic process of impurity impact ionization also leads to a strongly nonlinear curvature of the macroscopic (measured) current-voltage characteristic (with sometimes S-shaped negative differential resistance), the nonlinearity occurring just beyond the voltage corresponding to the critical electric field where the current increases by many orders of magnitude (typically, from a few $\mathrm{nA}$ in the pre-breakdown up to a few $\mathrm{mA}$ in the post-breakdown region). Under slight variation of distinct control parameters (electric field, magnetic field, and temperature in the range of some $10^{-6} \mathrm{~V} / \mathrm{cm}, 10^{-5} \mathrm{~T}$, and $10^{-3} \mathrm{~K}$, respectively) the resulting electric current flow exhibits a wide variety of spatial and temporal dissipative structures.

\section{Results}

The complex spatial behavior of our semiconductor system can be globally visualized by means of two-dimensional imaging of the current filament structures via low-temperature scanning electron microscopy. Current filaments represent high conducting channels embedded within a much lower conducting semiconductor medium [8]. Nucleation and growth of filamentary current patterns in the nonlinear postbreakdown regime are often accompanied by abrupt changes between different stable filament configurations via noisy current instabilities. Here a common phenomenon is that there exists a minimum as well as a maximum value of the filament current. On the one hand, with decreasing applied electric field, the filament size can not decrease continuously to zero but abruptly decays to zero at a distinct minimum diameter. On the other hand, with increasing applied electric field, a continuous growth of existing filaments takes place only up to a distinct maximum size beyond which an additional filament is nucleated. The ensuing multifilamentary current flow becomes more and more homogeneous if the semiconductor system further approaches its linear post-breakdown region at higher electric fields. On this way, the sample current usually exhibits pronounced jumps as a consequence of the abrupt nucleation (or annihilation) of individual filaments.

A comprehensive understanding of these experimental findings can be attained by a phenomenological theoretical description based on the electric power balance in the present nonequilibrium system [12]. The existence of a minimum diameter for current filaments is obvious from simple arguments balancing the power gain of moving hot charge carriers in the volume of the cylindrical filaments and the power losses accross the filament boundaries, thus giving rise to some critical surface to volume ratio. The maximum filament diameter was found to be given by the competing contributions of the power losses generated by the inhomogeneous current flow in the ohmic contact layers and those across the filament boundaries. In particular, minimization of the power sum of the contact dissipation and the filament surface dissipation could directly explain distinct bifurcation patterns observed experimentally. Here the current partially has split up into different filament branches (for an example of such complex multifilamentary current structure, see Fig. 2 of [3]). 
By additional help of both time-averaged and timeresolved current-voltage measurements, the underlying nonlinear physics of impurity impact ionization breakdown can be demonstrated to reveal critical phase transition behavior. Hereto, the temperature is varied as an appropriate thermodynamical control parameter, while distinct threshold voltages as well as the amplitude of self-generated oscillatory instabilities play the role of order parameters. Most important, we disclosed an upper bound for the onset of structure formation (first-order phase transition) and avalanche breakdown (second-order phase transition) at slightly different critical temperatures. The characteristic critical exponents evaluated indicate a scaling behavior consistent with the predictions of the Landau theory.
According to these preliminary results, low-temperature avalanche breakdown in extrinsic semiconductors promises to represent a fruitful testing ground for studying principles of nonequilibrium phase transitions and self-organized pattern-forming processes.

\section{Acknowledgements}

We thank Otto E. Rössler, Achim Kittel, Martina Knoop, Ulrich Buschmann, and Rudolf P. Huebener for discussions. Paper elaborated from a contribution presented at the 19th International Conference on Low Temperature Physics, Brighton, 16-22 August 1990.
[1] H. Haken, Advanced Synergetics, Springer, Berlin 1989.

[2] Y. Abe (ed.), Nonlinear and Chaotic Transport Phenomena in Semiconductors, special issue of Appl. Phys. A, Vol. 48, pp. 93-191, Springer, Berlin 1989.

[3] J. Peinke, J. Parisi, B. Röhricht, K. M. Mayer, U. Rau, and R. P. Huebener, Solid State Electron. 31, 817 (1988).

[4] K. M. Mayer, J. Parisi, and R. P. Huebener, Z. Phys. B, Condensed Matter 71, 171 (1988).

[5] K. M. Mayer, J. Parisi, J. Peinke, and R. P. Huebener, Physica D 32, 306 (1988).

[6] R. Stoop, J. Peinke, J. Parisi, B. Röhricht, and R. P. Huebener, Physica D 35, 425 (1989).
[7] U. Rau, K. M. Mayer, J. Parisi, J. Peinke, W. Clauß, and R. P. Huebener, Solid State Electron. 32, 1365 (1989).

[8] E. Schöll, Nonequilibrium Phase Transitions in Semiconductors, Springer, Berlin 1987.

[9] R. P. Huebener, J. Peinke, and J. Parisi, Appl. Phys. A 48, 107 (1989).

[10] J. Parisi, J. Peinke, B. Röhricht, U. Rau, M. Klein, and O. E. Rössler, Z. Naturforsch. 42 a, 655 (1987).

[11] J. Parisi, U. Rau, J. Peinke, and K. M. Mayer, Z. Phys. B, Condensed Matter 72, 225 (1988).

[12] K. M. Mayer, R. P. Huebener, and U. Rau, J. Appl. Phys. 67, 1412 (1990). 